\title{
Accurately Diagnosing COPD: A Clinical Challenge With Important Consequences
}

COPD is an irreversible chronic inflammatory process in the lungs that afflicts millions of patients around the world. ${ }^{1}$ Although COPD is usually considered primarily an inflammatory airway obstructive process, it also encompasses pathologic changes in smaller more distal membranous airways ("floppy," collapsing airways) and destruction of adjacent alveolar tissue (emphysema).

COPD is associated with significant morbidity and mortality and is responsible for yearly health care costs in the hundreds of billions of dollars. ${ }^{1}$ An accurate diagnosis of COPD is essential for multiple reasons: (1) Effective therapies exist but can be costly and often have risks, so they should be utilized only where they are needed. (2) Other diagnoses with similar respiratory symptoms exist and need to be ruled in or out for proper management. (3) COPD disease management programs need accurate inclusion/exclusion criteria for maximum effectiveness. (4) Clinical trials of new therapies and outcome studies of various practice patterns need to be carefully focused only on patients with COPD. (5) Public policies aimed at COPD prevention and control need the appropriate targets to address.

In this issue of RESPIRATORY CARE, Rice et $\mathrm{al}^{2}$ address the last reason given with a focus on an accurate COPD diagnosis to guide COPD readmission policies and penalties. ${ }^{2-4}$ Their primary concern is overdiagnosis; not surprisingly, COPD overdiagnosis is found to artificially inflate COPD readmission rates. This work, however, also brings into focus the challenges in determining a proper COPD diagnosis.

The classic definition of COPD is a purely clinical diagnosis: a chronic cough with sputum production for at least 3 months per year for 2 consecutive years. ${ }^{1}$ Unfortunately, using only clinical criteria to diagnose COPD ignores the reality that symptoms of COPD are nonspecific and can mimic many other cardio-respiratory disorders. Moreover, in sedentary elderly individuals, COPD symptoms may simply be written off as consequences of aging along with a seemingly benign "smokers cough." Clinician appreciation

The author has disclosed no conflicts of interest.

Correspondence: Neil R MacIntyre MD FAARC. E-mail: neil.macintyre@ duke.edu.

DOI: $10.4187 /$ respcare. 08741 of COPD signs/symptoms may also be poor, and the disease is often ignored or misclassified in patient problem lists. $^{5}$

A more widespread approach espoused by the Global

See the Original Study on Page 11

Obstructive Lung Disease (GOLD) consortium adds a spirometric assessment to establish a COPD diagnosis (ie, $\left.\mathrm{FEV}_{1} / \mathrm{FVC}<0.70\right){ }^{6}{ }^{6}$ However, the use of the spirometer as the sole diagnostic tool for COPD is also problematic for several reasons. First, the classic $\mathrm{FEV}_{1}$ and FVC measurements require a calibrated device, a trained tester, and a cooperative patient-a combination that is not always easy to obtain. Second, defining abnormal values for $\mathrm{FEV}_{1}, \mathrm{FVC}$, and $\mathrm{FEV}_{1} / \mathrm{FVC}$ has been controversial. The conventional statistical method defines abnormal as values below the fifth percentile of a distribution of values from a normal healthy population. ${ }^{7}$ This threshold is often termed the lower limit of normal and more accurately identifies airway abnormalities (especially in the young and old ends of the patient spectrum) better than arbitrary cut-points of $\mathrm{FEV}_{1} / \mathrm{FVC}<0.7$ and $\mathrm{FVC}$ and $\mathrm{FEV}_{1}$ values $<80 \%$ predicted. Third, $\mathrm{FEV}_{1} / \mathrm{FVC}$, the sentinel value to diagnose obstruction, can be both nonspecific and insensitive. Specifically, a low $\mathrm{FEV}_{1} / \mathrm{FVC}$ value can be present with other diseases impacting airway caliber and a normal $\mathrm{FEV}_{1} / \mathrm{FVC}$ ratio can be present in severe COPD limited to distal airways with emphysema. Fourth, $\mathrm{FEV}_{1}$ and FVC represent primarily properties of more proximal airways and expiratory muscle function. These spirometric indices are less able to detect abnormalities of smaller, more distal airways that are often involved early in the natural history of COPD. Finally, spirometry is incapable of detecting the presence of alveolar destruction (ie, emphysema) and loss of elastic recoil, which may be the only manifestations of COPD. Addressing this latter issue, the COPDgene clinical trials group has proposed adding an imaging component (eg, computed chest tomography) as a key component to establishing a COPD diagnosis. ${ }^{8}$

These diagnostic considerations impact the interpretation of the study by Rice et al. ${ }^{2}$ COPD overdiagnosis on an inpatient sample was considered present when a clinical COPD diagnosis was noted on a hospital admission but an 


\section{EDITORIALS}

accompanying spirometric measurement indicated a normal $\mathrm{FEV}_{1} / \mathrm{FVC}$ based on either the fixed 0.70 cut-point or the lower limit of normal. Importantly, patients with a COPD diagnosis but no spirometry were not included. Moreover, patients with an abnormal $\mathrm{FEV}_{1} / \mathrm{FVC}$ but no clinical COPD diagnosis were also excluded. Couple these issues with the imprecisions associated with a clinical diagnosis of COPD and the limited sensitivity and specificity of the spirogram, the specific numbers and percentages reported in the paper by Rice et $\mathrm{al}^{2}$ must be viewed with caution. This does not take away from their overall conclusion (ie, overdiagnosis almost certainly inflates readmission data) but limits estimating the magnitude of the effect.

So what are my take-home messages? First, recognize the impact of both under- and overdiagnosis of COPD on multiple aspects of COPD management, both on an individual level and on a population level. Second, be wary of using only clinical criteria to diagnose COPD. As noted above, signs and symptoms may be subtle, and many other diagnoses can act similarly, especially in the earlier stages. Third, respect the spirogram for what it can do but recognize the multiple pitfalls and limitations of the technique. Finally, is it time to consider adding new routine assessments to the evaluation of patients with suspected COPD? Examples might include pulmonary function tests such as forced oscillation analysis or diffusing capacity of the lung for carbon monoxide, which has particular appeal because it is widely available and sensitive to the presence of emphysema. ${ }^{9}$ Perhaps biomarker profiles that signify the presence/absence of COPD can be identified. Imaging approaches have considerable appeal, but routine computed tomography scanning, with its costs and risks, does not seem appropriate at the present time. Perhaps novel imaging such as ultrasound or electrical impedance tomography will emerge as useful in the future. At the end of the day, it is important that we recognize the importance of diagnosing COPD properly and continue to strive for better ways to do this.

Neil R MacIntyre

Duke University Medical Center Durham, North Carolina

\section{REFERENCES}

1. Kim V, Criner G. Chronic bronchitis and chronic obstructive pulmonary disease. Am J Respir Crit Care Med 2013;187(3):228-237.

2. Rice R, Han X, Wang X, Al-Jaghbeer M. COPD overdiagnosis and its effect on 30-day hospital readmission rates. Respir Care 2021;66 (1):11-17.

3. Jacobs J, Noyes K, Zhao J, Gibson W, Murphy TF, Sethi S, OchsBalcom HM. Early hospital readmissions after an acute exacerbation of chronic obstructive pulmonary disease in the Nationwide Readmissions Database. Ann Am Thorac Soc 2018;15(7):837-845.

4. Feemster LC, Au DH. Penalizing hospitals for COPD readmissions. Am J Respir Crit Care Med 2014;189(6):634-639.

5. Zaas D, Wise R, Wiener C. Airway obstruction is common but unsuspected in patients admitted to a general medicine service. Chest 2004;125(1):106-111.

6. Global Initiative for Chronic Obstructive Lung Disease (GOLD). Available at: https://goldcopd.org. Accessed December 14, 2020.

7. ATS Committee on Proficiency Standards for Pulmonary Function Laboratories. Recommendations for a standardized pulmonary function report - an official American Thoracic Society Technical Statement. Am J Respir Crit Care Med 2017;196(11):1463-1472.

8. Lowe KE, Regan EA, Anzueto A, Austin E, Austin JHM, Beaty TH, et al. COPDGene 2019: redefining the diagnosis of chronic obstructive pulmonary disease. Chronic Obstr Pulm Dis 2019;6(5):384-399.

9. Balasubramanian A, MacIntyre NR, Henderson RJ, Jensen RL, Kinney G, Stringer WW, et al. Diffusing capacity of carbon monoxide in assessment of COPD. Chest 2019;156(6):1111-1119. 